\title{
Synthesis of Some Imine, From Pyrazole -1- Carbaldehyde Compound
}

\author{
Natiq G. Ahmad ${ }^{1}$, Yassir S. Mohamad ${ }^{2 *}$ \\ ${ }^{1,2}$ Department of Chemistry, College of Education for Pure Science, University of Mosul, Mosul, Iraq \\ Email: ${ }^{1}$ natiq.ahmed@ uomosul.edu.iq ${ }^{2^{*}}$ yassir_chem71@uomosul.edu.iq
}

(Received December 24, 2012; Accepted April 03, 2013; Available online September 01, 2021)

\section{ABSTRACT}

In this paper the synthesis of $\mathrm{N}[(1 \mathrm{E})-1$ - ( 3,5 - disubstituted phenyl -4,5-dihydro-1H- pyrazol-1yl) ethylidene or methylidene] substituted aniline [13-15] [17-19] and 1,1-\{benzene-1,4- or -1,3- diylbis [nitrilo (E) methylylidene] \} bis( 4,5- dihydro -1H- pyrazole -3,5- disubstituted phenyl) [16,20] is reported. Substituted acetophenone was treated with substituted benzaldehyde to give chalcones ((2E)-1,3disubstituted phenyl prop-2-ene-1- one) [1-4], the chalcones was treated with hydrazine hydrate in the presence of formic or acetic acid ethanol to give 1-(3,5 -disubstituted phenyl-4,5- dihydro-1H- pyrazol-1yl) methanol or ethanone [5-12], then this products changed to imines through reaction with substituted aromatic aniline and sodium hydroxide in ethanol to give the substituted pyrazoles.

Also the substituted compounds [5-12] were converted to 1-\{(1E)-1-[2-(2,4-dinitrophenyl) hydrazinylidene] methyl or ethyl - 4,5- dihydro -1H- pyrazole-3,5-disubstituted phenyl [21-28] by reaction with 2,4-dinitrophenyl hydrazine in ethanol.

The synthetic compounds structure confirmed by IR., UV. Spectra and physical method.

Keywords: Imine, Pyrazole, Schiff bases.

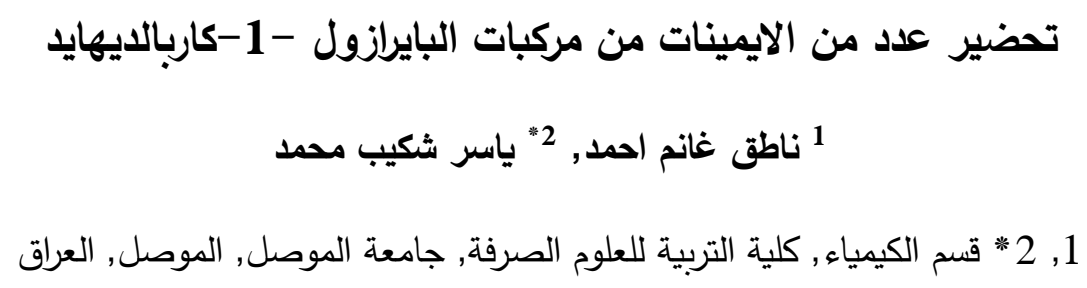




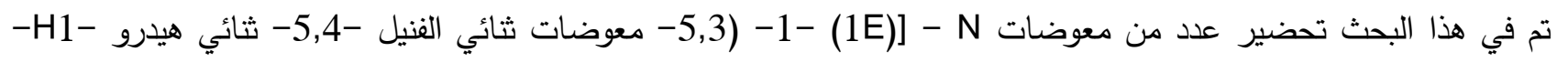

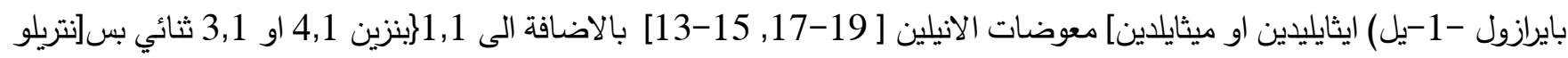
(E) ميثايلدين ] $\{$ بس (5,4- ثنائي هيدرو -H1- بايرازول - 5,3- ثنائي فنيل معوض) [16] و[ 20] حيث تم مفاعلة معوضات البنزلديهايد مع معوضات الاسيتوفينون في الوسط القاعدي لتحضير الجالكونات 3,1- ثنائي فنيل معوض بروبان -2-2-1انين - 1 - اون [1-14] ومن ثم مفاعلة الجالكونات المحضرة مع الهيدرازين المائي بوجود حامض الفورميك او حامض الخليك المذاب في الايثانول لأعطاء

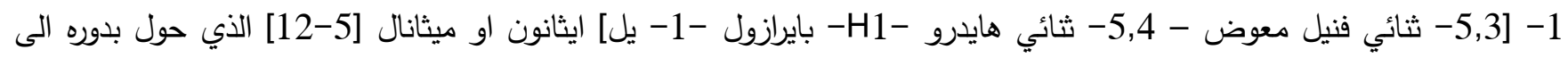
الايمين عن طريق مفاعلته مع امينات اروماتية في الايثانول بوسط قاعدي لأعطاء معوضات البايرازول. كما تم مفاعلة هذه المعوضات [5-12] مع 4,2- ثنائي نترو فنيل هيدرازين في الايثانول لأعطاء - -

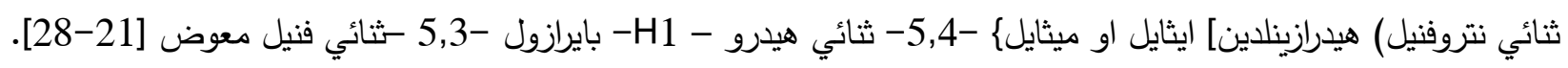
تم تثخيص المركبات المحضرة بواسطة طيف الأشعة تحت الحمراء والأشعة البنفسية والطرق الفيزياوية. الكلمات المفتاحية: ايمين, بايرازول, قواعد شيف.

\section{Introduction}

Schiff bases first reported by Schiff in 1864. The condensation of primary amines with carbonyl compounds are the main way to produce these compounds. The azomethine group with a general formula $\mathrm{RHC}=\mathrm{N}-\mathrm{R}^{\prime}$ was The common structural feature of these compounds, where R and R' are alkyl, aryl, cyclo., alkyl or heterocyclic groups which may be variously substituted. Compounds contain azomethine group also known as anils, imines ${ }^{(1)}$. Schiff bases can be synthesized from an aromatic amine and a carbonyl compound by nucleophilic addition forming a hemiaminal (contain hydroxyl and amine group ), then, by withdrawing of water to generate an imine. For instant, reaction, o-vanillin reacts with 4,4'diaminodiphenyl ether ${ }^{(2)}$.<smiles>COc1cccc(/C=N/c2ccc(Oc3ccc(/N=C/c4cc(C)cc(OC)c4O)cc3)cc2)c1O</smiles>

Schiff bases serve as a back bone for the synthesis of various heterocyclic compounds ${ }^{(3)}$ and Schiff base reactions are useful in making carbon-nitrogen bonds. Schiff bases look to count intermediates in a number of enzymatic reactions involving interaction of an enzyme with an amino or a 
carbonyl group of the substrate ${ }^{(4)}$. A sequence of transition metal complexes of, Co (II), Ni (II), and Cu (II) have been synthesized from the Schiff base derived from 4-aminoantipyrine 3-hydroxy-4nitrobenzaldehyde and o-phenylenediamine. The structural activity of the above metal complexes shows that $\mathrm{Cu}, \mathrm{Ni}$ and $\mathrm{Co}$ complexes cleave DNA through redox chemistry ${ }^{(5)}$. Also Schiff Bases are insecticides, fluorescent brighteners, antitumor and other biological activities ${ }^{(6-10)}$.

\section{Experimental}

Melting points apparatuses electro thermal 9300 engineering LTD in addition, IR spectrum was recorded infrared spectrophotometer model tensor 27 Bruker Co, Germany, using KBr discs. The U.V spectrum was recorded on UV-Visible Shimadzu 1601spectrophotometer using ethanol/ethyl acetate 1:1 as a solvent. Chemicals were purchased from Fluka and TCI Ltd.

chalcones ((2E)-1,3-disubstituted phenyl prop-2-ene-1- one $)(1-4)^{(11,12)}$.

A mixture of substituted benzaldehyde $(0.1 \mathrm{~mol})$ and substituted acetophenone $(0.1 \mathrm{~mol})$ were dissolved in ethanol then located in cold bath after that, the reaction pot stirred for $3 \mathrm{~h}$. Using $(10 \% \mathrm{NaOH})$ (cold solution) drop by drop. The mixture was gone to stand for 2hours, the solid products was filtered then dried and used ethanol for recrystallizing to afford chalcones. Table (1) scheduled physical properties and spectral data.

\section{1-(3,5-disubstituted phenyl -4,5- dihydro -1H- pyrazol -1- yl) methanol or ethanone [5-12 ${ }^{(13-15)}$.}

A solution of hydrazine hydrate (30) $\mathrm{ml}$ in (15) $\mathrm{ml}$ of ethanol was added drop wise with cooling to a solution of (15) $\mathrm{ml}$ of chalcons and (20) $\mathrm{ml}$ of acetic acid. The resultant refluxed with stirring for 24 hours. After that, the reaction left to cool to room temperature and pieces of ice through in a solution with cooling to induce the precipitation. The crud product recrystallized with ethyl acetate. Table (2) listed physical properties and spectral data.

\section{N[(1E)-1- ( 3,5 - disubstituted phenyl-4,5- dihydro-1H- pyrazol-1- yl) ethylidene or methylidene]} substituted aniline [13-15,17-19] and 1,1-\{benzene- 1,4- or -1,3- diylbis [nitrilo (E) methylylidene]\} bis( 4,5- dihydro -1H- pyrazole -3,5- disubstituted phenyl) $[16,20]^{(16,2)}$.

pyrazoles $(0.01 \mathrm{~mol})$ [5-12] was mixed with $(0.01 \mathrm{~mol})$ of the proper substituted aniline(in case synthesized compound [16] and [20] we must use (0.02 mol) of proper pyrazoles) in $(15 \mathrm{~mL})$ of the ethanol and the combination was stirred. A solution of sodium hydroxid( $0.4 \mathrm{~g}$ in $15 \mathrm{~mL}$ ethanol) was added 
gradually to the reaction during a period of 30 minutes and the combination was refluxed for $2 \mathrm{~h}$ at $60 \mathrm{c}^{\circ}$. with continual stirring. The final mixture leaved for about $2 \mathrm{~h}$., poured on to crushed ice,dried, recrystallized from ethanol-water. Table (3) recorded physical properties and spectral data.

1-\{(1E)-1- [2-(2,4- dinitrophenyl) hydrazinylidene] methyl or ethyl $\}$ - 4,5- dihydro -1H- pyrazole 3,5- disubstituted phenyl [21-28 ${ }^{(17)}$.

In a test tube put $(0.05 \mathrm{~g})$ of compounds $[5-12]$ in $(0.5 \mathrm{~mL})$ ethanol then $(5 \mathrm{ml})$ of the saturated solution of 2,4-dinitrophenyl- hydrazine in $(2 \mathrm{M}) \mathrm{HCl}$, the mixture was shacked for 10 second. The solid products (yellow- red) filtered and recrystallized by ethyl acetate-water. Table(4). listed physical properties and spectral data.

\section{Results and Discussion}

In this paper the synthesis of $\mathrm{N}[(1 \mathrm{E})-1-($ 3,5 - disubstituted phenyl-4,5- dihydro-1H-pyrazol -1yl) ethylidene or methylidene] substituted aniline [13-15,17-19] and 1,1-\{benzene- 1,4- or -1,3- diylbis [nitrilo (E) methylylidene] \} bis (4,5- dihydro -1H-pyrazole -3,5-disubstituted phenyl) [16,20] is reported (Scheme1).

Substituted acetophenone was treated with substituted benzaldehyde afford chalcones [1-4]. The IR. spectra $^{(18)}$ show absorption at $\mathbf{v}(1657-1685) \mathrm{cm}^{-}(\mathrm{C}=\mathrm{O})$ and $\boldsymbol{v}(1602-1658) \mathrm{cm}^{-}(\mathrm{C}=\mathrm{C}), \quad \lambda_{\max }(331-$ 400)nm. (table1)

Table (1): physical properties and spectral of compouns [1-4]

\begin{tabular}{|c|c|c|c|c|c|c|c|c|c|}
\hline \multirow[b]{2}{*}{$\begin{array}{l}\text { Com. } \\
\text { No. }\end{array}$} & \multirow[b]{2}{*}{$\begin{array}{l}\text { Yield } \\
(\%)\end{array}$} & \multirow[b]{2}{*}{ m.p. $\left({ }^{\circ} \mathrm{C}\right)$} & \multirow[b]{2}{*}{ Colour } & \multirow{2}{*}{$\begin{array}{c}\text { U.V } \\
\lambda \text { max } \\
\text { in ethanol/ } \\
\text { ethyl } \\
\text { acetate } 1: 1\end{array}$} & \multicolumn{5}{|c|}{$\begin{array}{c}\text { I.R v } \\
\mathrm{cm}^{-1}(\mathrm{KBr})\end{array}$} \\
\hline & & & & & $\mathrm{C}=\mathbf{O}$ & $\begin{array}{c}\mathbf{C - H} \\
\text { aromatic }\end{array}$ & $\mathrm{C}=\mathrm{C}$ & $\begin{array}{l}\text { C-O- } \\
\text { C } \\
\text { Sym. } \\
\text { Assy } \\
\text { m. }\end{array}$ & $\begin{array}{c}\mathrm{NO}_{2} \\
\text { sym. } \\
\text { assym. }\end{array}$ \\
\hline 1 & 85 & $159-161$ & Yellow & 357 & 1658 & 3030 & 1608 & - & $\begin{array}{l}1337, \\
1515 \\
\end{array}$ \\
\hline 2 & 87 & 207-209 & Red & 354 & 1668 & 3050 & 1611 & - & $\begin{array}{c}1317, \\
1519\end{array}$ \\
\hline 3 & 58 & $61-63$ & Yellow & 331 & 1657 & 2959 & 1602 & $\begin{array}{l}1048 \\
1267\end{array}$ & - \\
\hline 4 & 65 & $202-204$ & Brown & 400 & 1685 & 3002 & 1658 & $\begin{array}{l}1041 \\
1248\end{array}$ & $\begin{array}{c}1348, \\
1528\end{array}$ \\
\hline
\end{tabular}


The chalcones were treated with formic or acetic acid and hydrazine in ethanol to give 1-( 3,5disubstituted phenyl-4,5-dihydro -1H- pyrazol-1- yl) methanal or ethanone (5-12). The IR shifts ${ }^{(13)}$ of $^{-1}$ compounds [5-12] show absorption at $\mathbf{v}(1649-1698) \mathrm{cm}^{-}(\mathrm{C}=\mathrm{O})$ and $\boldsymbol{v}(1606-1657) \mathrm{cm}^{-}(\mathrm{C}=\mathrm{N}), \lambda_{\max }(331-$ 360)nm. (Table 2).

Table (2): physical properties and spectral of compounds [5-12]

\begin{tabular}{|c|c|c|c|c|c|c|c|c|c|}
\hline \multirow[b]{2}{*}{$\begin{array}{c}\text { Com. } \\
\text { No. }\end{array}$} & \multirow[b]{2}{*}{ Yield(\%) } & \multirow[b]{2}{*}{ m.p. $\left({ }^{\circ} \mathrm{C}\right)$} & \multirow[b]{2}{*}{ Colour } & \multirow{2}{*}{$\begin{array}{c}\mathrm{U.V} \\
\lambda \text { max } \\
\text { in ethanol/ } \\
\text { ethyl acetate } \\
1: 1\end{array}$} & \multicolumn{5}{|c|}{$\begin{array}{c}\text { I.R v } \\
\mathrm{cm}^{-1}(\mathrm{KBr})\end{array}$} \\
\hline & & & & & $\mathrm{C}=\mathrm{O}$ & $\begin{array}{c}\mathbf{C}-\mathbf{H} \\
\text { aromatic }\end{array}$ & $\mathbf{C}=\mathbf{N}$ & $\begin{array}{c}\text { C-O-C } \\
\text { Sym. } \\
\text { Assym } \\
.\end{array}$ & $\begin{array}{c}\mathrm{NO}_{2} \\
\text { sym. } \\
\text { assym. }\end{array}$ \\
\hline 5 & 60 & $115-116$ & Orange & 332 & 1680 & 3076 & 1657 & - & $\begin{array}{l}1345 \\
1514 \\
\end{array}$ \\
\hline 6 & 75 & 144-146 & Orange & 332 & 1676 & 3081 & 1630 & - & $\begin{array}{l}1318 \\
1522 \\
\end{array}$ \\
\hline 7 & 58 & $105-107$ & Yellow & 338 & 1661 & 3051 & 1607 & $\begin{array}{l}1055 \\
1272 \\
\end{array}$ & - \\
\hline 8 & 64 & 264-266 & Brown & 329 & 1697 & 3045 & 1620 & $\begin{array}{l}1053 \\
1250 \\
\end{array}$ & $\begin{array}{l}1320 \\
1528 \\
\end{array}$ \\
\hline 9 & 63 & $151-153$ & Yellow & 329 & 1649 & 3041 & 1610 & - & $\begin{array}{l}1350 \\
1518 \\
\end{array}$ \\
\hline 10 & 69 & $99-101$ & Yellow & 333 & 1653 & 3050 & 1606 & - & $\begin{array}{l}1348 \\
1528 \\
\end{array}$ \\
\hline 11 & 71 & $83-82$ & Yellow & 329 & 1699 & 3044 & 1652 & $\begin{array}{l}1047 \\
1285 \\
\end{array}$ & - \\
\hline 12 & 55 & $112-114$ & Orange & 331 & 1698 & 3043 & 1654 & $\begin{array}{l}1045 \\
1285 \\
\end{array}$ & $\begin{array}{l}1314 \\
1530\end{array}$ \\
\hline
\end{tabular}

The pyrazols were changed to imines by reaction with substituted aromatic aniline in presence of sodium hydroxide in ethanol to give the compound[13-20]. The IR $\operatorname{spectra}^{(16)}$ of [13-20] show absorption at $\boldsymbol{v}(1605-1681) \mathrm{cm}^{-}$for $(\mathrm{C}=\mathrm{N})$ and $\boldsymbol{v}(1161-1180) \mathrm{cm}^{-}$for $(\mathrm{N}-\mathrm{N}), \lambda_{\max }(329-333) \mathrm{nm}$. (Table 3). 
Table (3): physical properties and spectral of compounds [13-20]

\begin{tabular}{|c|c|c|c|c|c|c|c|c|c|}
\hline \multirow[b]{2}{*}{$\begin{array}{c}\text { Com. } \\
\text { No. }\end{array}$} & \multirow[b]{2}{*}{$\begin{array}{l}\text { Yield } \\
(\%)\end{array}$} & \multirow[b]{2}{*}{ m.p. $\left({ }^{\circ} \mathrm{C}\right)$} & \multirow[b]{2}{*}{ Colour } & \multirow{2}{*}{$\begin{array}{c}\text { U.V } \\
\lambda \text { max } \\
\text { in ethanol/ ethyl } \\
\text { acetate } 1: 1\end{array}$} & \multicolumn{5}{|c|}{$\begin{array}{c}\text { I.R v } \\
\mathrm{cm}^{-1}(\mathrm{KBr})\end{array}$} \\
\hline & & & & & $\mathrm{C}=\mathrm{N}$ & $\begin{array}{c}\mathbf{C}-\mathbf{H} \\
\text { aromatic }\end{array}$ & C-N & $\begin{array}{c}\text { C-O-C } \\
\text { Sym. } \\
\text { Assym. }\end{array}$ & $\begin{array}{c}\mathrm{NO}_{2} \\
\text { sym. } \\
\text { assym }\end{array}$ \\
\hline 13 & 83 & $292-294$ & Yellow-red & 333 & 1650 & 3014 & 1180 & - & $\begin{array}{l}1335 \\
1520\end{array}$ \\
\hline 14 & 78 & $119-121$ & Brown & 333 & 1674 & 3080 & 1178 & - & $\begin{array}{l}1309 \\
1521\end{array}$ \\
\hline 15 & 80 & $289-291$ & Yellow & 329 & 1647 & 2995 & 1168 & $\begin{array}{l}1051 \\
1240 \\
\end{array}$ & - \\
\hline 16 & 86 & $249-251$ & Brown & 329 & 1681 & 3040 & 1168 & $\begin{array}{l}1194 \\
1254\end{array}$ & $\begin{array}{l}1317 \\
1529\end{array}$ \\
\hline 17 & 83 & $250-253$ & Yellow & 329 & 1647 & 3041 & 1180 & - & $\begin{array}{l}1334 \\
1521\end{array}$ \\
\hline 18 & 79 & $154-156$ & Brown & 332 & 1628 & 3035 & 1179 & - & $\begin{array}{l}1302 \\
1528\end{array}$ \\
\hline 19 & 78 & 104-106 & Yellow & 332 & 1605 & 3058 & 1178 & $\begin{array}{l}1051 \\
1260\end{array}$ & - \\
\hline 20 & 75 & $142-144$ & Brown & 329 & 1651 & 3083 & 1161 & $\begin{array}{l}1040 \\
1258\end{array}$ & $\begin{array}{l}1315 \\
1529\end{array}$ \\
\hline
\end{tabular}

The presence of carbonyl group in the pyrazol ring have been proved by using the chemical reagent 2,4- dinitrophenylhydrazine which yield the hydrazone product [21-28] which identified through I.R spectra $^{(19-20)}$ which indicate the disappear of carbonyl group and the appearance of imines group at $\mathbf{v}(1602-$ $1675) \mathrm{cm}^{-}$for $(\mathrm{C}=\mathrm{N})$; v(1317-1348) $\mathrm{cm}^{-}$symmetric and $\boldsymbol{v}(1515-1530) \mathrm{cm}^{-}$asymmetric for $\left(\mathrm{NO}_{2}\right)$ and v(3421-3448) $\mathrm{cm}^{-}$for $(\mathrm{N}-\mathrm{H}), \lambda_{\max }(236-329) \mathrm{nm}$.(table 4).

Table(4): physical properties and spectral of compounds [21-28]

\begin{tabular}{|c|c|c|c|c|c|c|c|c|}
\hline \multirow[b]{2}{*}{$\begin{array}{l}\text { Com. } \\
\text { No. }\end{array}$} & \multirow[b]{2}{*}{ Yield $(\%)$} & \multirow[b]{2}{*}{ m.p. $\left({ }^{\circ} \mathrm{C}\right)$} & \multirow[b]{2}{*}{ Colour } & \multirow{2}{*}{$\begin{array}{c}\mathrm{U} . \mathrm{V} \\
\lambda \text { max } \\
\text { in ethanol/ } \\
\text { ethyl } \\
\text { acetate 1:1 }\end{array}$} & \multicolumn{4}{|c|}{$\begin{array}{c}\text { I.R v } \\
\mathrm{cm}^{-1}(\mathrm{KBr})\end{array}$} \\
\hline & & & & & $\mathrm{C}=\mathrm{N}$ & N-H & $\begin{array}{c}\mathrm{NO}_{2} \\
\text { sym. } \\
\text { assym }\end{array}$ & $\begin{array}{l}\text { C-O-C } \\
\text { Sym. } \\
\text { Assym. }\end{array}$ \\
\hline 21 & 85 & $118-120$ & Red & 329 & 1664 & 3448 & $\begin{array}{l}1339 \\
1515 \\
\end{array}$ & - \\
\hline 22 & 78 & $150-149$ & Yellow-red & 329 & 1675 & 3443 & $\begin{array}{l}1319 \\
1520\end{array}$ & - \\
\hline 23 & 79 & $108-110$ & Orange & 329 & 1640 & 3435 & $\begin{array}{l}1320 \\
1525\end{array}$ & $\begin{array}{l}1055 \\
1250\end{array}$ \\
\hline 24 & 77 & $135-137$ & Orange & 329 & 1621 & 3422 & $\begin{array}{l}1317 \\
1522\end{array}$ & $\begin{array}{l}1057 \\
1280\end{array}$ \\
\hline 25 & 82 & $191-193$ & Orange & 329 & 1602 & 3423 & $\begin{array}{l}1334 \\
1520\end{array}$ & - \\
\hline 26 & 80 & $260-262$ & Yellow-red & 329 & 1617 & 3422 & $\begin{array}{l}1347 \\
1519\end{array}$ & - \\
\hline 27 & 73 & $61-63$ & Orange & 332 & 1620 & 3420 & $\begin{array}{l}1325 \\
1520\end{array}$ & $\begin{array}{l}1045 \\
1247\end{array}$ \\
\hline 28 & 80 & 106-108 & Yellow & 332 & 1618 & 3421 & $\begin{array}{l}1348 \\
1518\end{array}$ & $\begin{array}{l}1041 \\
1267\end{array}$ \\
\hline
\end{tabular}


Journal of Education and Science (ISSN 1812-125X), Vol: 30, No: 4, 2021 (220-229)

Table(5): groups of compound [1-12 ]

\begin{tabular}{|c|c|c|c|c|c|c|c|c|c|}
\hline No. & $\mathbf{X}$ & $\mathbf{Y}$ & $\mathbf{Z}$ & $\mathbf{R}$ & $\mathbf{N o}$. & $\mathbf{X}$ & $\mathbf{Y}$ & $\mathbf{Z}$ & $\mathbf{R}$ \\
\hline $\mathbf{1}$ & $\mathrm{NO}_{2}$ & $\mathrm{H}$ & $\mathrm{H}$ & - & $\mathbf{9}$ & $\begin{array}{c}\mathrm{NO} \\
2\end{array}$ & $\mathrm{H}$ & $\mathrm{H}$ & $\mathrm{CH}_{3}$ \\
\hline $\mathbf{2}$ & $\mathrm{H}$ & $\mathrm{H}$ & $\mathrm{NO}_{2}$ & - & $\mathbf{1 0}$ & $\mathrm{H}$ & $\mathrm{H}$ & $\mathrm{NO}_{2}$ & $\mathrm{CH}_{3}$ \\
\hline $\mathbf{3}$ & $\mathrm{H}$ & $\mathrm{OCH}_{3}$ & $\mathrm{H}$ & - & $\mathbf{1 1}$ & $\mathrm{H}$ & $\mathrm{OCH}_{3}$ & $\mathrm{H}$ & $\mathrm{CH}_{3}$ \\
\hline $\mathbf{4}$ & $\mathrm{H}$ & $\mathrm{OCH}_{3}$ & $\mathrm{NO}_{2}$ & - & $\mathbf{1 2}$ & $\mathrm{H}$ & $\mathrm{OCH}_{3}$ & $\mathrm{NO}_{2}$ & $\mathrm{CH}_{3}$ \\
\hline $\mathbf{5}$ & $\mathrm{NO}_{2}$ & $\mathrm{H}$ & $\mathrm{H}$ & $\mathrm{H}$ & & & & & \\
\hline $\mathbf{6}$ & $\mathrm{H}$ & $\mathrm{H}$ & $\mathrm{NO}_{2}$ & $\mathrm{H}$ & & & & & \\
\hline $\mathbf{7}$ & $\mathrm{H}$ & $\mathrm{OCH}_{3}$ & $\mathrm{H}$ & $\mathrm{H}$ & & & & & \\
\hline $\mathbf{8}$ & $\mathrm{H}$ & $\mathrm{OCH}_{3}$ & $\mathrm{NO}_{2}$ & $\mathrm{H}$ & & & & & \\
\hline
\end{tabular}

Table (6): groups of compound[13 - 28 ]

\begin{tabular}{|c|c|c|c|c|c|c|c|c|c|c|c|c|}
\hline No. & $\mathbf{X}$ & $\mathbf{Y}$ & $\mathbf{Z}$ & $\mathbf{R}$ & $\mathbf{A}$ & $\mathbf{B}$ & $\mathbf{C}$ & $\mathbf{N o}$ & $\mathbf{X}$ & $\mathbf{Y}$ & $\mathbf{Z}$ & $\mathbf{R}$ \\
\hline $\mathbf{1 3}$ & $\mathrm{NO}_{2}$ & $\mathrm{H}$ & $\mathrm{H}$ & $\mathrm{H}$ & $\mathrm{H}$ & $\mathrm{CH}_{3}$ & $\mathrm{H}$ & $\mathbf{2 1}$ & $\mathrm{NO}_{2}$ & $\mathrm{H}$ & $\mathrm{H}$ & $\mathrm{H}$ \\
\hline $\mathbf{1 4}$ & $\mathrm{H}$ & $\mathrm{H}$ & $\mathrm{NO}_{2}$ & $\mathrm{H}$ & $\mathrm{H}$ & $\mathrm{NO}_{2}$ & $\mathrm{H}$ & $\mathbf{2 2}$ & $\mathrm{H}$ & $\mathrm{H}$ & $\mathrm{NO}_{2}$ & $\mathrm{H}$ \\
\hline $\mathbf{1 5}$ & $\mathrm{H}$ & $\mathrm{OCH}_{3}$ & $\mathrm{H}$ & $\mathrm{H}$ & $\mathrm{CH}_{3}$ & $\mathrm{H}$ & $\mathrm{H}$ & $\mathbf{2 3}$ & $\mathrm{H}$ & $\mathrm{OCH}_{3}$ & $\mathrm{H}$ & $\mathrm{H}$ \\
\hline $\mathbf{1 6}$ & $\mathrm{H}$ & $\mathrm{OCH}_{3}$ & $\mathrm{NO}_{2}$ & $\mathrm{H}$ & $\mathrm{H}$ & $\mathrm{NH}_{2}$ & $\mathrm{H}$ & $\mathbf{2 4}$ & $\mathrm{H}$ & $\mathrm{OCH}_{3}$ & $\mathrm{NO}_{2}$ & $\mathrm{H}$ \\
\hline $\mathbf{1 7}$ & $\mathrm{NO}_{2}$ & $\mathrm{H}$ & $\mathrm{H}$ & $\mathrm{CH}_{3}$ & $\mathrm{H}$ & $\mathrm{CH}_{3}$ & $\mathrm{H}$ & $\mathbf{2 5}$ & $\mathrm{NO}_{2}$ & $\mathrm{H}$ & $\mathrm{H}$ & $\mathrm{CH}_{3}$ \\
\hline $\mathbf{1 8}$ & $\mathrm{H}$ & $\mathrm{H}$ & $\mathrm{NO}_{2}$ & $\mathrm{CH}_{3}$ & $\mathrm{H}$ & $\mathrm{NO}_{2}$ & $\mathrm{H}$ & $\mathbf{2 6}$ & $\mathrm{H}$ & $\mathrm{H}$ & $\mathrm{NO}_{2}$ & $\mathrm{CH}_{3}$ \\
\hline $\mathbf{1 9}$ & $\mathrm{H}$ & $\mathrm{OCH}_{3}$ & $\mathrm{H}$ & $\mathrm{CH}_{3}$ & $\mathrm{CH}$ & $\mathrm{H}$ & $\mathrm{H}$ & $\mathbf{2 7}$ & $\mathrm{H}$ & $\mathrm{OCH}_{3}$ & $\mathrm{H}$ & $\mathrm{CH}_{3}$ \\
\hline $\mathbf{2 0}$ & $\mathrm{H}$ & $\mathrm{OCH}_{3}$ & $\mathrm{NO}_{2}$ & $\mathrm{CH}_{3}$ & $\mathrm{H}$ & $\mathrm{H}$ & $\mathrm{NH}_{2}$ & $\mathbf{2 8}$ & $\mathrm{H}$ & $\mathrm{OCH}_{3}$ & $\mathrm{NO}_{2}$ & $\mathrm{CH}_{3}$ \\
\hline
\end{tabular}


<smiles>[X]c1ccc(C=O)c([X])c1</smiles><smiles>[X]c1cccc(C2=NNC(c3ccc([X])cc3[Y])C2)c1</smiles><smiles>[R]C(C)=NNc1ccc([N+](=O)[O-])cc1[N+](=O)[O-]</smiles><smiles>[O+][Os]</smiles><smiles>[C+]c1cccc(C2=NC(/N=C/c3cccc(/N=C\N4N=C(c5cccc([N+](=O)[O-])c5)CC4c4ccccc4OC)c3)C(c3ccccc3OC)C2)c1</smiles>
[20]<smiles>[Z]c1cccc(C(C)=O)c1</smiles>

2,4-dinitrophenylhydrazine<smiles>C#CC#C</smiles><smiles>C=CN1N=C(c2cccc([N+](=O)[O-])c2)CC1c1ccccc1OC</smiles>

Scheme (1)

Note: see table $(5,6)$ for substituted group

\section{References}

1- Muhammad A. A., Karamat M. and Abdul W., International Conference on Chemistry and Chemical Process, vol.10:p.1 (2011).

2- Vhanale B.T., Deshmukh N.J. and Shinde A.T. ; Synthesis, characterization, spectroscopic studies and biological evaluation of Schiff bases derived from 1-hydroxy-2-acetonapthanone; Heliyon, $\underline{\text { V. 5, Issue }}$ $\underline{11},(2019)$.

3- Santosh K., Niranjan M. S., Chaluvaraju K. C., Jamakhandi C. M. and Dayanand K.; Synthesis and Antimicrobial Study of Some Schiff Bases of Sulfonamides Journal of Current Pharmaceutical Research, 1, 39-42 (2010). 
4- Malik A., Sher K. S., Wajid R., Zonera H. A. and Muhammad I.; Cytotoxic, antibacterial activity and physico-chemical properties of some acid catalyzed Schiff bases; African Journal of Biotechnology Vol. 10 (2), p. 209-213, (2011).

5- Raman N., Jdhaveethu R. and Sakthivel A.; Synthesis, spectral characterization of Schiff base transition metal complexes: DNA cleavage and antimicrobial activity studies; J. Chem. Sci., Vol. 119, No., pp. 303310, (2007).

6- Seniz K. and Zuhal F.; Synthesis of Schiff bases by condensation of hetarylcarboxaldehydes with $p$ phenetidine; fur chemie, 121, 525-528 (1990).

7- Satyanarayana V. S. V., Sreevani P., Amaravadi S., and Vijayakumar V.; ARKIVOC (xvii) 221-233 (2008). Arkivoc (xvii) 221-233 (2008).

8- Chandramouli, Shivanand M. R., Thakar B. N. and Udupi R. H.; Synthesis and biological screening of certain new triazole schiff bases and their derivatives bearing substituted benzothiazole moiety; Journal of Chemical and Pharmaceutical Research, 4(2):1151-1159, (2012).

9- Mehmet G.U., Mehmet S,Ismet B.; Synthesis, characterization, and antimicrobial activity of a new pyrimidine Schiff base and its $\mathrm{Cu}(\mathrm{II}), \mathrm{Ni}(\mathrm{II}), \mathrm{Co}(\mathrm{II}), \mathrm{Pt}(\mathrm{II})$, and Pd(II) complexes; Turk. J. Chem., 36, 189 - 200, (2012).

10- Wadher S. J. , Puranik M. P., Karande N. A. and Yeole P.G.; Synthesis and Biological Evaluation of Schiff base of Dapsone and their derivative as Antimicrobial agents; International Journal of PharmTech Research, Vol.1,No.1, p. 22-33 ( 2009).

11- Patil D.Y. and Rachmale,I.; synthesis and antimicrobial activity of some chalcone derivatives and their coppercomplexes; International Journal of Pharmaceutical Sciences and Research, Vol. 3(3): 901908, (2012).

12- Koneni V. S., Manoj K., Abdhesh K.; A novel route to synthesis of flavones from salicylaldehyde and acetophenone derivatives; Tetrahedron Letters, 53(18), 2355-2359(2012).

13-Zangade S. B., Shinde A. T., Vibhute A. Y., Vibhute Y. B., An Improved Synthesis And Biological Evaluation Of Some New 4,5-dihydro-pyrazole-1-Carbaldehyde Derivatives Pakistan Journal of Chemistry, Volume: 2, P. 18-23, (2012).

14- Ching-Chun T., Shuo-En T., Sin-Min L. and Fung F. W.; One-Pot Acid-Promoted Synthesis of 6Aminopyrazolopyrimidines from $1 H$-Pyrazol-5-yl- $N, N$-dimethylformamidines or 5-Amino-1 $H$-pyrazole4-carbaldehydes with Cyanamide; J. Org. Chem. , 84, 24, 16157-16170, (2019).

15- Mohd J., Ozai A., Farah N., Md. Jahangir A. and Prwaiz A.; Current status of pyrazole and its biological activities; J. Pharm Bioallied Sci., 8(1) p 2-17, (2016).

16- Azzouz A.S.P., Ali R.T.; Synthesis of Schiff Bases Derived from Benzaldehyde and Salicylaldehyde with Some Amino Acids by a New Develop Method; National Journal of Chemistry, Vol. 37, P. 158-168, (2010).

17- Karen Feinstein ; Guid to spectroscopic identification of organic compounds ; Boca Raton, CRC Press (2018).

18- Suvitha S., Siddig I. A., Mohammed Ali A. and Syam M.; synthesis of Chalcones with Anticancer Activities; Molecules 17(6), P.6179-6195(2012). 
19- Brand, Izabella; Application of polarization modulation infrared reflection absorption spectroscopy in electrochemistry; Springer international publishing, (2020).

20- Asmaa B. S., Adil A. F., Majeed Y. Y.; Synthesis and Antimicrobial Evaluation of Some New Organic Tellurium Compounds Based on Pyrazole Derivatives; Molecules, 25, (15), 3439, (2020). 